\title{
Safety of extending screening intervals beyond five years in cervical screening programmes with testing for high risk human papillomavirus: 14 year follow-up of population based randomised cohort in the Netherlands
}

\section{(9) $\mathbb{( 1 )}$ OPEN ACCESS}

It has come to our attention that there is an error in Figure 1 of this Research paper (BMJ 2016;355:i4924, doi:10.1136/bmj. i4924). The two text boxes "Cyt positive and HPV positive (n=369)" should read "Cyt positive and HPV negative (n=369)." The paper has since been corrected. 